\title{
Repair Measures for a Seismically Retrofitted Steel Bridge Experiencing Distortion-Induced Fatigue Cracks: Numerical Study
}

\author{
Mehdi Motaleb ${ }^{1(凶)}$, Nick Duong $^{1}$, Ahmed Ibrahim $^{2}$, Will Lindquist ${ }^{3}$, \\ and Riyadh Hindi ${ }^{4}$ \\ ${ }^{1}$ Parks College of Aviation, Engineering and Technology, Saint Louis \\ University, St. Louis, MO 63103, USA \\ motalebm@slu.edu \\ ${ }^{2}$ Department of Civil Engineering, College of Engineering, University of Idaho, \\ Moscow, USA \\ aibrahim@uidaho.edu \\ ${ }^{3}$ William Jewell College, Liberty, MO 64068, USA \\ lindquistw@william.jewell.edu \\ ${ }^{4}$ Graduate Education and Research, Parks College of Aviation, Engineering and \\ Technology, Saint Louis University, St. Louis, MO 63103, USA \\ rhindi@slu.edu
}

\begin{abstract}
This paper studies distortion-induced fatigue cracks in a 1960's era design Missouri Department of Transportation (MoDOT) welded plate girder bridge that developed cracks in the web-gap region shortly after completion of a comprehensive seismic retrofit. Finite Element analysis (FEA) was conducted in order to investigate the cause of cracking and to recommend appropriate repair measures for the bridge. The results indicated that the high stress concentrations were principally a result of replacing K-type diaphragms with stiffer cross-diaphragms as part of the seismic retrofit. Two conventional repair measures in addition to two innovative repair measures were evaluated. The first repair strategy provided a positive connection between the connection plate and the girder flange (referred to as the top-angle repair). The second repair created a 203-mm (8-in.) slot where one-sixth of the connection plate depth is cut from the web (slot-repair) resulting in a softening of the web gap.

Two additional innovative repair measures are also proposed. The first consisted of adding a vertical angle section to either side of the transverse connection plate (middle-angle repair). This repair method stiffened the web-gap region, and as a result, restrained the web-gap region from deforming out of plane. The other technique intended to reduce stress levels by softening the web-gap region as well as distributing lateral forces, transferred by cross-frames, over a wider area of the web (slot-angle repair). The proposed innovative repair measures focused on ease of installation compared to more conventional options while they also appear able to reduce the distortion-induced stresses well below the constant amplitude fatigue threshold (CAFT).
\end{abstract}




\section{Introduction}

The Federal Highway Administration (FHWA) required an immediate review of all double-deck bridge structures in the United States following the collapse of a double-deck bridge in the 1989 Loma Prieta Earthquake. The Missouri Department of Transportation (MoDOT) initiated a seismic retrofit project for the U.S. 40/I-64 double deck bridge complex in St. Louis, Missouri after the structural review of this 1960's era design indicated that many of the substructure and superstructure elements did not have adequate capacity required by current seismic criteria (Finke 2006; Yen et al. 2001). The seismic retrofit strategy was adopted based on the criteria described in the FHWA Seismic Retrofitting Manual for Highway Bridges (Buckle et al. 2006). Shortly after completion of the seismic retrofit, MoDOT officials began to identify several cracks in the longitudinal girder webs originating at retrofit locations. These cracks were observed to initiate in the unstiffened region of the cross-frame-to-girder connections in an area called the web gap. Cracks originating in this region are likely the result of secondary stresses resulting from out-of-plane distortion in the unstiffened web-gap region and account for approximately $90 \%$ of all fatigue cracking (Connor and Fisher 2006).

This paper investigates first, the cause of cracking in the interior girder web-gap region, and second, determines the effectiveness and behavior of four different repair techniques through finite element (FE) modeling. Four repair options, including two conventional and two innovative repairs were evaluated using the FE analysis for their potential to arrest existing cracks and to prevent new cracks from initiating. The stresses in the web-gap region of the repaired bridge were compared with original bridge, prior to the identification of cracking, and with the corresponding locations of the seismically retrofitted bridge. The goal of the repair strategies was to reduce web-gap stresses to an acceptable level recommended by (AASHTO 2012) in order to avoid the initiation of additional fatigue cracks.

\section{Case Study Bridge Information}

The section under consideration is from the upper deck and consists of four spans with a total length of approximately $97.5 \mathrm{~m}$ (320 ft.) from a larger double-deck bridge complex approximately $2.41 \mathrm{~km}$ (1.5 miles) in length that carries more than 93,000 vehicles per day (MoDOT 2013). The superstructure is comprised of a cast-in-place reinforced concrete upper deck composite with longitudinal multi-steel plate girders that are supported by cross girders seated on reinforced concrete columns as shown in Fig. 1.

The cross girders are simply supported whereas the longitudinal girders are continuous between expansion joints. A plan view of the original bridge is shown in Fig. 2 (a) showing the cross girders label according to their number relative to the entire complex.

The original intermediate cross frames (diaphragms) consist of L $3 \frac{1}{2} \times 3 \frac{1}{2} \times 5 / 16$ angles for the top chords and L3 $\times 2 \frac{1}{2} \times 5 / 16$ angles for the remaining members as shown in Fig. 2(b). 


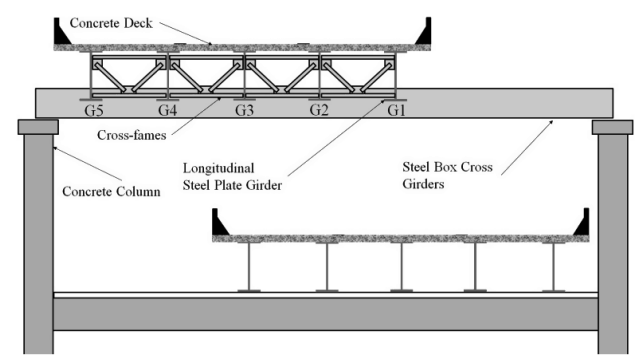

Fig. 1. Double-deck bridge complex cross view

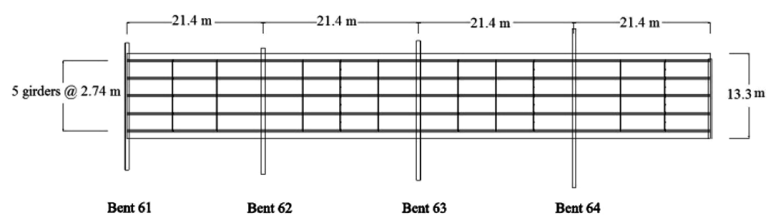

(a)

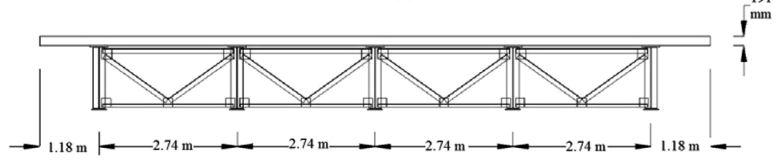

(b)

Fig. 2. Original bridge (a) plan-view and (b) cross-view

Earthquake forces and ductility requirements were not well understood at the time this structure was designed, and as a result, a detailed seismic analysis was not required nor completed. However, a subsequent design review found that structural modifications were essential for the upper superstructure and supporting columns to resist new horizontal shear in both the longitudinal and transverse direction as well as displacements in both directions. Thus, as a part of the seismic retrofit, the existing " $\mathrm{K}$ " diaphragms were replaced with " $\times$ " diaphragms consisting of $2 L 4 \times 4 \times 3 / 8$ double angles.

During routine inspection within approximately one year following the completion of the seismic retrofit, MoDOT inspectors identified several cracks in the webs of the longitudinal plate girders. Most of the cracks were found in the three interior girders at the location of the cross-girder retrofit and new " $x$ " bracing as shown in Fig. 3 . The majority of the cracks were initially oriented in the longitudinal direction originating from the toe of the web-to-flange weld or the weld at the end of the stiffener plate (connection plate for the cross frames) with some turning downward and progressing deeper into the web. Figure 4 shows a perspective view of a typical crack that has been formed in a web-gap region of the bridge. 


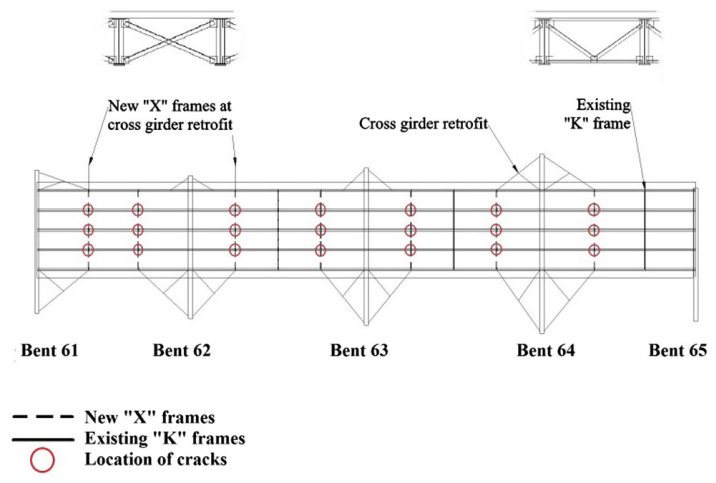

Fig. 3. Seismic retrofitted bridge plan-view

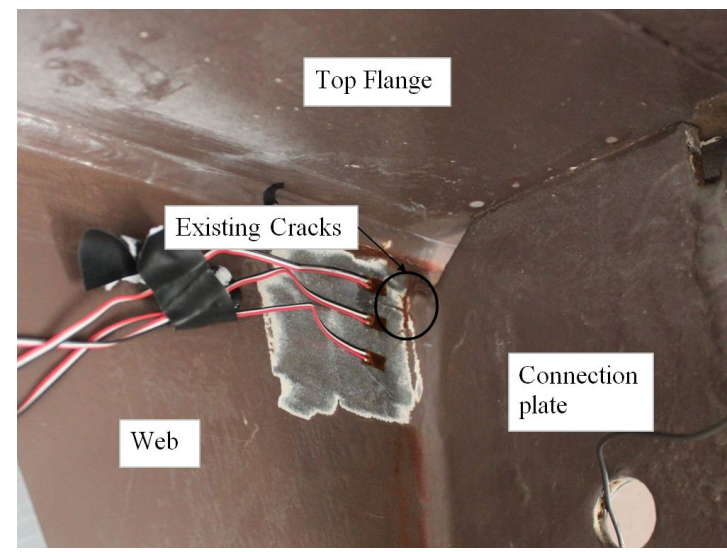

Fig. 4. an existing crack in the web gap region of girder 2

\section{Finite Element Modeling}

In order to minimize the computational cost of the finite element (FE) analysis, a dual-level modeling method was adopted. A macro model was developed containing four spans of the bridge between two expansion joints as shown in Fig. 5(a). This model was used to determine the critical transverse and longitudinal location for the truck. However, rigorous mesh sensitivity was not conducted on Macro model, so that the localized behavior in the web-gap region cannot be used for comparison purposes. Therefore, in order to capture the out-of-plane distortion in the web-gap region, a more sophisticated FE model (micro-model) that covers a much smaller part of the bridge was developed using Abaqus/CAE, as shown in Fig. 5(b). All boundary conditions for this micro-model were imported from the macro-model. Tie interactions were used to connect the top flange and concrete deck to create full composite action, and surface-to-surface contacts were used between bolted parts enabling them to slide 


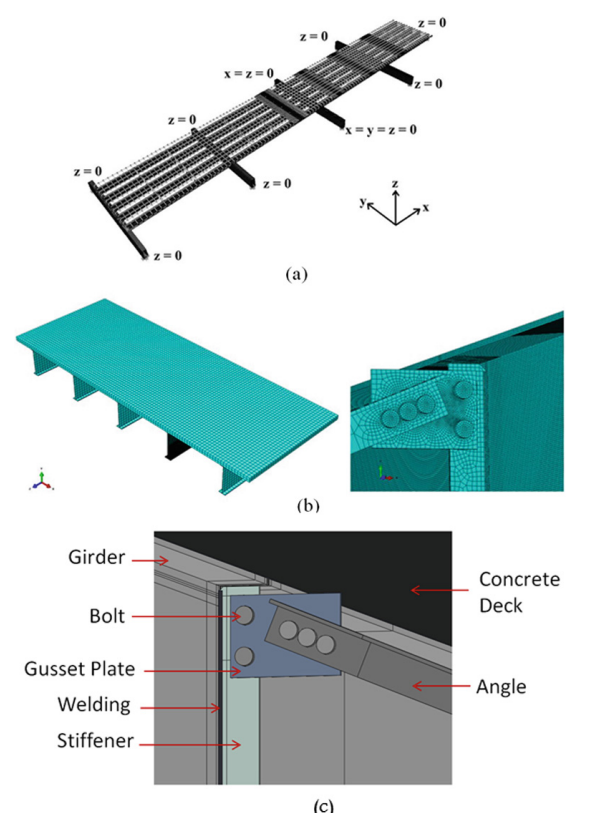

(c)

Fig. 5. FE models (a) Macro-model in SAP2000, (b) micro-model in Abaqus and (c) detailed connection in the web-gap region

depending on bolt and bolt-hole deformation. Figure 5(c) shows a view of one longitudinal girder with the connection plate, gusset plate, bolts, and cross-frame.

All the members in FE models were created using 3D solid eight-node elements with three translational degrees of freedom and reduced integration (C3D8R). Mesh sensitivity was determined by performing analyses with various element sizes for G2, which was the primary girder of interest for the micro-model. The hotspot stress and peak stress in the web-flange joint was determined from this girder and used to compare the efficiency of each repair technique. An element size of $5.08 \mathrm{~mm}(0.2 \mathrm{in}$.) was used for G2 as the hotspot stress (described in Stress Analysis section) in the web-gap region converged to a constant value, as shown in Fig. 6. A coarser mesh of $120 \mathrm{~mm}$ (4.75 in.) was used for the other girders away from the location of interest. The models contained approximately one million elements, but varied slightly between FE models based on the repair option evaluated.

The pre-tensioning forces in the bolts, was modeled by applying individual axial tensile loads to the bolts in the first step of the analysis so that each 25.4-mm (1-in.) diameter bolt carried a specified pretension force of $227 \mathrm{kN}$ (51 kips) as defined by AASHTO (2012). The bolts were modeled explicitly with the pretensioning force applied to the middle of the shank. Surface contact interactions were defined for each bolt and the surrounding members. The welds were idealized using right triangles and modeled using eight-node solid elements.

In order to determine the critical load location in the longitudinal and transverse directions, three possibilities were examined including: (1) a moving load applied to 


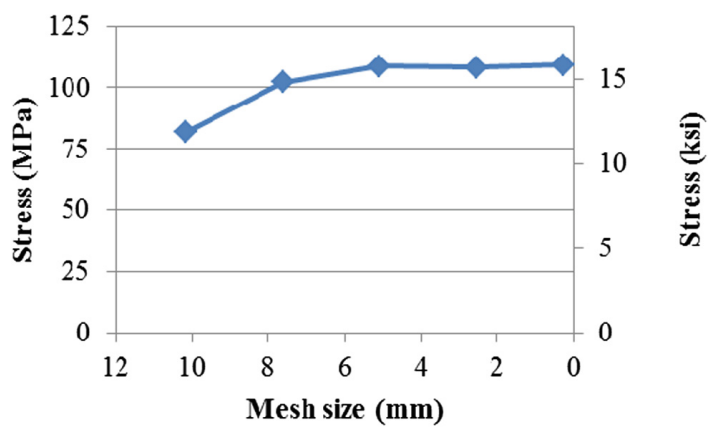

Fig. 6. Mesh sensitivity analysis

the bridge in the right lane, (2) a moving load applied in the middle lane, and (3) a moving load applied in the left lane. These models were run using SAP2000 as reported by Lindquist et al. (2015).

In addition, the critical longitudinal location of the truck was estimated with influence lines for a continuous beam representing the four-span composite steel girders. The truck load was then applied as a static loading on the macro-model to maximize stress at the web gap location and compared with the maximum stress due to the moving load. This static analysis provided the required boundary conditions (displacements and rotations) that were applied to the micro-model in order to simulate the truck loading.

\subsection{Stress Analysis}

In this study, the hotspot stress analysis approach was used to determine stresses for the fatigue analysis. A hotspot is the term used to refer to the critical point in a structure where fatigue cracking can be expected (Niemi 1995). In this approach, the fatigue strength is generally based on the value of stress near the point of crack initiation. The hotspot stress at welded connection was determined using reference points located away from the weld, as shown in Fig. 7. The reference paths (based on the thickness of the web $\mathrm{t}$ ) including references points were located at distances of $0.5 \mathrm{t}$ and $1.5 \mathrm{t}$ measured perpendicular from the weld toe, as shown in Fig. 7. The reference point locations were based on AASHTO (2012) recommendations for fatigue design verification of orthotropic steel bridge decks. The hotspot stress $\mathrm{f}_{\mathrm{hss}}$ was determined using Eq. 1 (AASHTO 2012).

$$
f_{h s s}=1.5 f_{0.5}-0.5 f_{1.5}
$$

where:

$f_{0.5}=$ Principal stress at a distance of $0.5 \mathrm{t}$ from the weld toe

$f_{1.5}=$ Principal stress at a distance of $1.5 \mathrm{t}$ from the weld toe. 


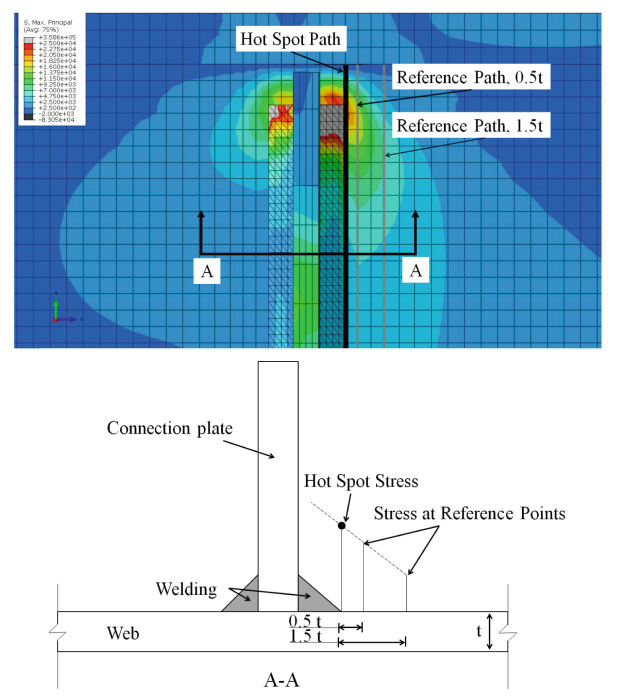

Fig. 7. Hotspot path

In order to evaluate the stress at the end of the vertical fillet of the stiffener-to-web joint, the stresses for all nodes along the weld were determined and the maximum stress along this path was selected as the hotspot stress. A similar procedure was used by (Hartman 2013; Richardson et al. 2012) to provide a consistent comparison between models even as the stress distribution in the web-gap changed between FE models.

However, the hotspot method was not able to predict the value of stresses corresponding to the longitudinal cracks along the horizontal fillet weld of the web-to-flange joint. The reason was that two fields of stresses from two sources overlapped (i.e., stiffener-to-web joint stress field and web-to-flange joint stress field). Therefore, stresses on the longitudinal reference paths could not be used to determine the stress at the longitudinal weld toe due to potential interference resulting from stresses emanating from the web-to-flange joint. For purposes of this study, the peak stress along the horizontal fillet weld on the web surface was adopted for comparisons. This peak stress from the web-to-flange joint was used to compare the efficiency of different repair options in reducing the stress in the web-to-flange joint area.

\section{FE Results and Repair Investigations}

FE analysis showed that the distortion-induced stresses developed in the top web gaps for the seismically retrofitted bridge were considerably higher than those for the original bridge. The hotspot stress increased from $24.8 \mathrm{MPa}(3.60 \mathrm{ksi})$ to $94.5 \mathrm{MPa}$ $(13.7 \mathrm{ksi})$ when the seismic retrofitting elements were added to the original bridge. This explained the field observation that shortly after completion of the seismic retrofit a large number of web gap cracks initiated in the longitudinal girder webs originating at retrofit locations. Based on the results obtained from FE analysis the main cause for 
those cracks was replacement of the " $\mathrm{K}$ "-type diaphragms with the much stiffer cross diaphragms. The new cross diaphragms are $2 \mathrm{~L} 4 \times 4 \times 3 / 8$ angles (compared to single L3 $\times 2 \frac{1}{2} \times 5 / 16$ angles) which are capable of transferring a much large force between girders. These forces generated the out-of-plane distortion in the web-gap region with the potential to result in fatigue cracking since the increased stress exceeds the CAFT for a category C detail (AASHTO 2012).

This type of crack usually forms in planes parallel to the primary stresses from loading the girder, and at initial stage has negligible effect on flexural behavior of girders. However, if the cracks do not be repaired at that stage, the cracks grow and can potentially turn perpendicular to the applied stress from external loads. Therefore, a solution to limit the out-of-plane distortions and corresponding stresses is crucial for the long-term stability of the bridge.

Several field and analytical investigations have been conducted to evaluate the effectiveness and feasibility of repairing members damaged by distortional stresses (Connor and Fisher 2006; Fisher and Keating 1989; Richardson et al. 2012; Shifferaw and Fanous 2013; Zhao and Roddis 2000). In general, these repair options fall into one of two categories including (1) increasing the stiffness of the web-gap region by providing a positive attachment between the connection plate and the longitudinal girder flanges, or (2) increasing the flexibility of the region in order to reduce the stress concentrations. Variations of both of these repair strategies are evaluated as part of this study. The efficacy of four repair options aimed to reduce web-gap stresses in G2 when the middle lane is investigated. The purpose of this analysis is to identify potential repair options that should be validated with a field investigation upon completion of a pilot repair. The first repair strategy provided a positive connection between the connection plate and the girder flange (referred to as the top-angle repair). The second repair created a 203-mm (8-in.) slot where one-sixth of the connection plate depth is cut from the web (slot-repair) resulting in a softening of the web gap.

Two additional innovative repair measures are also proposed. The first consisted of adding a vertical angle section to either side of the transverse connection plate (middle-angle repair). This repair method stiffened the web-gap region, and as a result, restrained the web-gap region from deforming out of plane. This option is cost-effective, easy-to-install, and does not significantly interrupt traffic. The other technique intended to reduce stress levels by softening the web-gap region as well as distributing lateral forces, transferred by cross-frames, over a wider area of the web (slot-angle repair). The four repair strategies are shown in Fig. 8. Figure 9 shows the hotspot stresses for the four repair methods compared to the unrepaired bridge (cracked). Also, to evaluate stresses on the other side of the web, opposite of where the hotspot stress was captured, a 1-m (40-in.) path along the horizontal fillet weld of the web-to-flange joint was defined for each repair technique. Principal stresses along this path are shown in Fig. 10. The principal stresses reached their peak at the intersection of the web and connection plate for most of the repair options. Details for each repair technique are discussed as follows. 


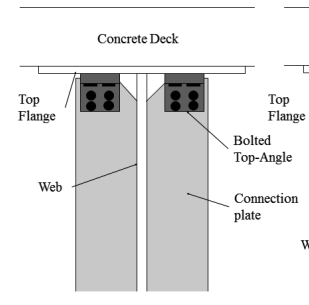

(a)

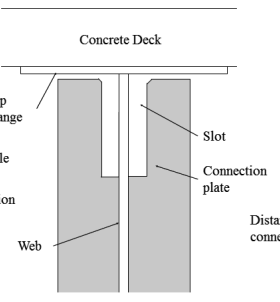

(b)

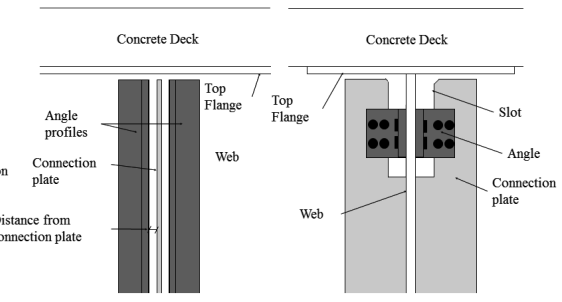

(c)

(d)

Fig. 8. Retrofit options evaluated by FE models including (a) top-angle repair, (b) slot repair, (c) middle-angle repair, and (d) slot-angle repair

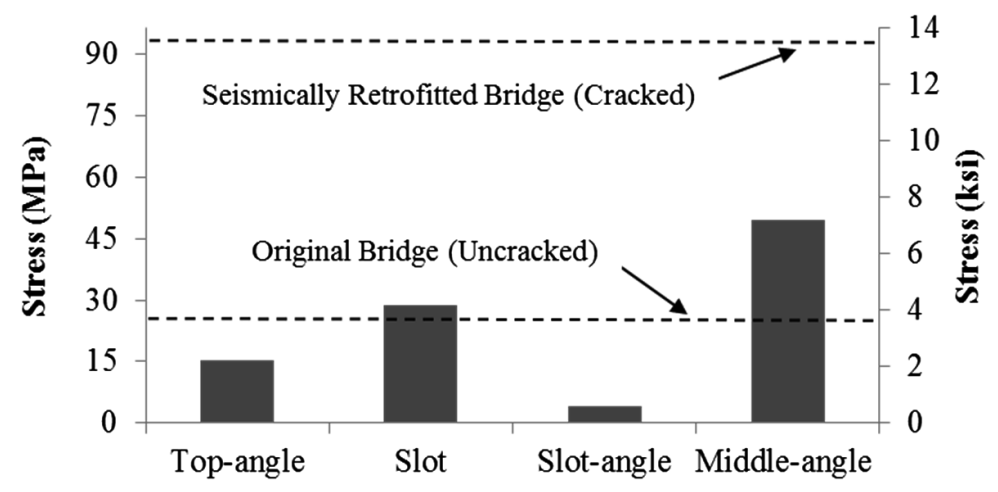

Fig. 9. Comparison of web gap stress values before and after repair

\subsection{Top-Angle Option}

A traditional repair for web-gap distortion is to stiffen the area by positively attaching the connection plate to the girder flange (Fisher 1990; Zhao and Roddis 2004). This attachment restrains web-gap distortion, and as a result, is able to greatly lower out-of-plane displacement in the web-gap region. In order to evaluate the performance of this repair, a L4 $\times 4 \times 3 / 8$ angle was used to attach the connection plate and top flange. Attachment was provided by modeling bolts which were preloaded to create the minimum required bolt tension specified in AASHTO (2012). As shown in Fig. 9, the bolted attachment resulted in a significant reduction in the hot-spot stress through a reduction in the relative displacement between the girder flange and connection plate. The hotspot stress in the web-gap region dropped from $94.5 \mathrm{MPa}(13.7 \mathrm{ksi})$ to 15.4 $\mathrm{MPa}(2.24 \mathrm{ksi}), 84 \%$ reduction, for the bolted connection which is considerably below the CAFT of $68 \mathrm{MPa}(10 \mathrm{ksi})$. Also, this repair option reduced the peak value of stress along the horizontal fillet weld of the web-to-flange joint to $20.9 \mathrm{MPa}(3.03 \mathrm{ksi})$ from $118 \mathrm{MPa}(17.1 \mathrm{ksi}), 82 \%$ reduction.

However, many in-service steel bridges have significant construction limitations due to the desire to limit traffic disruptions, making the top-angle repair solution difficult because in most cases, a portion of the concrete deck must be removed in order 
to tighten the bolts to the top flange. In many cases, there is also not enough room to attach the top-angle in the desired location due to existing bolts connecting the gusset and connection (stiffener) plates. Another limitation for this technique is that it can only be implemented if net section fracture does not control the strength of the girder since holes must be drilled into the girder flange.

\subsection{Slot Option}

The second measure investigated to repair web-gap cracking is to soften the area by creating a slot at the top of the connection plate thereby making a softer web-gap. This method was first used in 1980 for the retrofit of the Des Moines (Polk County) Bridge (Fisher 1984). Dexter and Ocel (2013) recommend that the length of the new web-gap be one-sixth the web depth but no greater than $381 \mathrm{~mm}$ (15.0 in.). Thus, a cut dimension of $203 \mathrm{~mm}$ ( $8 \mathrm{in}$.) was utilized for this repair, as shown schematically in Fig. 8(b). The slot repair method resulted in a significant stress reduction in the web-gap region by shifting the stress concentration location down and providing a more flexible web-gap region. The hot spot stress developed in this model is $28.7 \mathrm{MPa}$ (4.16 ksi) compared to $94.5 \mathrm{MPa}(13.7 \mathrm{ksi})$ corresponding to a stress reduction of $70 \%$. The slot repair resulted in a considerable reduction in the peak stress along the horizontal fillet weld of the web-to-flange joint with a stress of only 13.0 MPa $(1.89 \mathrm{ksi})$ an $89 \%$ reduction from the post retrofitted bridge. Although this method is an efficient option in reducing web-gap stresses, it has been somewhat less effective in practice. Fatigue cracks were found shortly after this type of retrofit was completed in a Lexington Avenue Bridge (Dexter and Ocel 2013).

\subsection{Middle-Angle Repair Option}

An innovative repair option to stiffen the web-gap region is investigated which does not have the same limitations as the top-angle option. In this method, L4 $\times 4 \times 3 / 8$ angles were placed on the surface of the web and $25.4 \mathrm{~mm}(1 \mathrm{in}$.) from the connection plate which provided enough space to accommodate existing bolts. Four angle sections were placed on either side of the connection plate on both sides of the web. This technique reduced the hotspot stress to $49.6 \mathrm{MPa}(7.19 \mathrm{ksi})$ which corresponds to a $48 \%$ stress reduction (moderately below the CAFT of $10 \mathrm{ksi}$ ). In addition, the peak stress on the opposite side of the web at the web-to-flange joint was reduced to $59.0 \mathrm{MPa}(8.55 \mathrm{ksi})$ which represents a $50 \%$ reduction. Also, as Fig. 10 shows, there is a moderate reduction in the peak value of stress along the horizontal fillet weld of the web-to-flange joint compared to the cracked bridge (bridge before repair).

This method can be implemented without creating a new fatigue sensitive area and can provide more convenient installation as the clearance between the section and connection plate allows the exiting bolts to remain in place. 


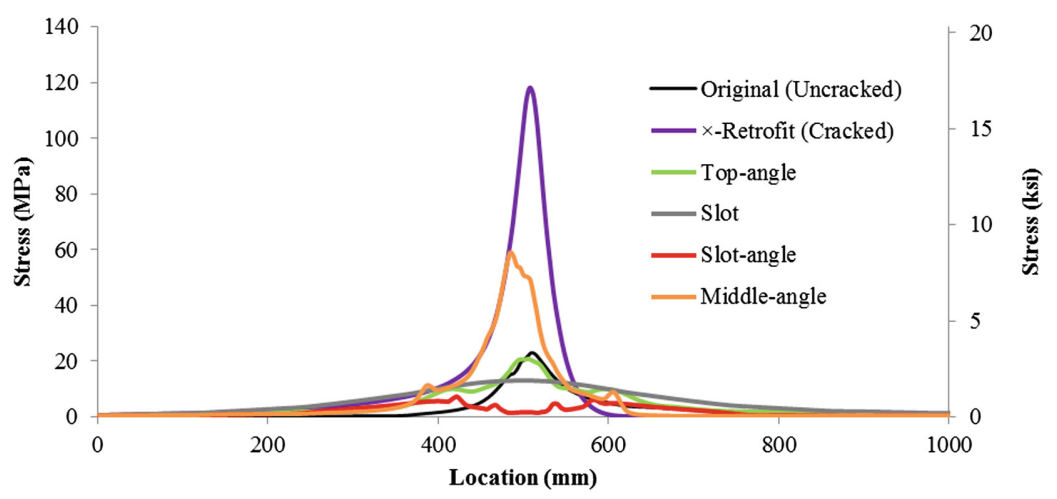

Fig. 10. Principal stress along the horizontal fillet weld of the web-to-flange joint on the web surface

\subsection{Slot-Angle Repair Option}

The slot repair showed acceptable performance in stress reduction as it covers one-sixth of the web depth. However, this technique was performed to soften the web-gap of a Kansas Department of Transportation welded plate girder bridge and it failed to prevent cracks from reinitiating at some of the repair locations (Zhao and Roddis 2007). Therefore, eliminating the stress concentrations at the connection plate-web joint end can be an effective way to prevent re-initiation of cracks at the location of the new stress concentration. The fourth repair option evaluated in this study, as shown in Fig. 8(d), involves cutting the connection plate and using a L4 $\times 4 \times 3 / 8$ angle to attach the disconnected part of connection plate to the web.

This technique softens the web-gap and distributes the load transferred from the diaphragm to a wider area. Also, it restrains large deformations at the top of the connection plate that is disconnected from web. As a result, the hot spot stress drops more than what was observed for slot technique alone. The same slot dimensions were used for this repair and each $\mathrm{L} 4 \times 4 \times 3 / 8$ angle attaches connection plate to web using eight bolts, as shown in Fig. 8(d). The hot spot stress was reduced by $96 \%$ to $3.99 \mathrm{MPa}$ $(0.579 \mathrm{ksi})$. Figure 9 shows a significant stress reduction within the new web gap as a result of this repair option. Also, the value of peak stress on the opposite side of the web at the web-to-flange joint was reduced to $7.31 \mathrm{MPa}$ (1.06 ksi) from $118 \mathrm{MPa}$ (17.1 ksi) corresponding to a $94 \%$ reduction. There was no considerable localized stress distribution for the slot-angle repair option.

This new technique eliminated hot spot stress concentrations while bolted angles on either side of the stiffener provide enough rigidity to resist lateral deformation of the connection plate. The results showed that the slot-angle method defines a new larger load path to transfer the driving force from the diaphragm to the web reducing the stress concentrations below the critical stress required to initiate fatigue cracks. 


\section{Conclusions}

The FE studies were carried out on a double-deck multi-girder steel bridge experienced distortion induced fatigue cracking after the completion of a seismic retrofit. Four repair options were recommended to reduce stresses in the web-gap region so that both crack initiation and crack growth could be prevented. The numerical study presented herein indicated the following:

- The high stress concentrations in the web-gap region of the retrofitted bridge was found large enough to lead to fatigue cracking after only a limited number of cycles.

- The FE results showed that distortion-induced stresses developed at the top web gaps for the seismically retrofitted bridge were much higher than those for the original bridge ( $281 \%$ increase in hotspot stress).

- The main cause of cracking was due to the replacement of the " $\mathrm{K}$ "-type diaphragms with much stiffer cross diaphragms resulting in stresses above the CAFT defined by AASHTO (2012).

- The top-angle repair method showed a significant stress reduction in the web-gap region by $84 \%$. Also, this repair option reduced the peak value of stress along the horizontal fillet weld of the web-to-flange joint by $82 \%$.

- The middle-angle repair option was a new technique that did not have the same construction limitations as the top-angle repair while reducing the hotspot stress by $48 \%$ and below the constant amplitude fatigue threshold required for preventing fatigue crack initiation and growth.

- Among the repair options considered in this paper, the slot-angle technique showed the best performance in reducing both hot-spot stress and peak stress along the horizontal fillet weld of the web-to-flange joint.

- The slot-angle repair option was also evaluated as an alternative to the slot option. The addition of angles adjacent to the web may overcome some limitations present with the traditional slot repair.

Acknowledgments. Support for the research described in this paper was provided by the Missouri Department of Transportation.

\section{References}

AASHTO: LRFD Bridge Design Specifications. Washington, DC (2012)

Buckle, I., Friedland, I., Mander, J., Martin, G., Nutt, R., Power, M.: Seismic retrofitting manual for highway structures: part 1-bridges (2006)

Connor, R.J., Fisher, J.W.: Identifying effective and ineffective retrofits for distortion fatigue cracking in steel bridges using field instrumentation. J. Bridge Eng. 11, 745-752 (2006)

Dexter, R.J., Ocel, J.M.: Manual for Repair and Retrofit of Fatigue Cracks in Steel Bridges (2013)

Finke, J.: Construction of Seismic Retrofit Strategies of the US 40/I-64 Double Deck Bridge Complex (2006)

Fisher, J.W.: Fatigue and fracture in steel bridges. Case Studies (1984) 
Fisher, J.W., American Association of State Highway and Transportation Officials, and United States, Federal Highway Administration: Distortion-Induced Fatigue Cracking in Steel Bridges. Transportation Research Board, National Research Council (1990)

Fisher, J.W., Keating, P.B.: Distortion-induced fatigue cracking of bridge details with web gaps. J. Constr. Steel Res. 12(3), 215-228 (1989)

Hartman, A.: Analytical and Experimental Investigation for Distortion-Induced Fatigue in Steel Bridges (2013)

Lindquist, W., Ibrahim, A., Tung, Y., Motaleb, M., Tobias, D., Hindi, R.: Distortion-induced fatigue cracking in a seismically retrofitted steel bridge. J. Perform. Constr. Facil. 30(4), 04015068 (2015)

MoDOT: St. Louis District Traffic Volume and Commercial Vehicle Count Map. Missouri Department of Transportation (2013)

Niemi, E.: Stress Determination for Fatigue Analysis of Welded Components. Woodhead Publishing, Cambridge (1995)

Richardson, T., Alemdar, F., Bennett, C., Matamoros, A., Rolfe, S.: retrofit measures for distortion-induced fatigue. Modern Steel Constr. 52(4), 32-34 (2012)

Shifferaw, Y., Fanous, F.S.: Field testing and finite element analysis of steel bridge retrofits for distortion-induced fatigue. Eng. Struct. 49, 385-395 (2013)

Yen, W.P., O'Fallon, J.D., Cooper, J.D., Ger, J.J.: FHWA bridge seismic retrofit manual and its applications in Missouri highway bridges. In: Structures 2001@sA Structural Engineering Odyssey, pp. 1-8. ASCE (2001)

Zhao, Y., Roddis, W.K.: Fatigue crack investigation for the Arkansas river bridge in Hutchinson, Kansas. Constr. Build. Mater. 14(5), 287-295 (2000)

Zhao, Y., Roddis, W.K.: Fatigue Prone Steel Bridge Details: Investigation and Recommended Repairs. Kansas Department of Transportation (2004)

Zhao, Y., Roddis, W.M.K.: Fatigue behavior and retrofit investigation of distortion-induced web gap cracking. J. Bridge Eng. 12, 737-745 (2007) 